\title{
1 Habitat determines convergent evolution of cephalic horns in vipers
}

2 Evolution of horns in vipers

3 Theo Busschau $^{1}$ \& Stephane Boissinot ${ }^{1}$

$4 \quad{ }^{1}$ New York University Abu Dhabi, Saadiyat Island, Abu Dhabi, United Arab Emirates

5

6 Corresponding authors:

7 tb2613@nyu.edu (T.B.)

8 sb5272@nyu.edu (S.B.) 


\section{Abstract}

10 Phenotypic convergence of traits in similar environments can provide insights into the

11 evolutionary processes shaping trait evolution. Among squamate reptiles, horn-like cephalic

12 appendages have evolved under various selective pressures, including selection for defence,

13 crypsis or sexual selection. Yet, among snakes, particularly vipers, the functional and

14 evolutionary significance of horns are unknown. We used a comparative phylogenetic

15 approach with habitat and diet data on 263 viper taxa to shed light on the selective pressures

underlying horn evolution in vipers. We detected significant correlations with habitat but not

diet. The relative positions of horns are ecologically divergent in that supranasal horns are positively correlated with terrestrial forest habitats while supraocular horns are negatively correlated with terrestrial forest habitats and associated with arboreal or sparsely vegetated habitats. Multiple independent origins of supranasal or supraocular horns in similar habitats provide evidence of adaptive convergence. Comparisons with other snake lineages suggest that cephalic appendages may have evolved under selection for crypsis in ambush foraging snakes.

24 Keywords: ambush foraging - ancestral state reconstruction - cephalic appendages - crypsis 
27 Understanding the adaptive significance of phenotypic variation is an important goal in evolutionary biology. The convergence of seemingly similar traits shared among distantly related species may provide insights into the underlying evolutionary processes (Mahler et al., 2017). If a trait evolved independently and is associated with a similar environment or ecological niche, it is likely to be adaptive to that environment (Losos, 2011).

Among squamate reptiles, horn-like cephalic appendages evolved under various selective pressures. For example, in horned lizards, Phrynosoma spp., cranial horns evolved as defensive weapons against predation (Bergmann \& Berk, 2012). In chameleons, horns are used in male combat, territorial displays, or to persuade females during copulation and are thus under strong sexual selection (Karsten et al., 2009; Stuart-fox, 2013). Rostral appendages in agamids are thought to be under sexual selection and natural selection for crypsis (Karunarathna et al., 2020).

In comparison to other squamates, cephalic appendages are rare among extant ophidians and their functional significance still largely unknown. The only study investigating the function of cephalic appendages in snakes is on the aquatic Erpeton tentaculatum Lacépède, 1800 (Homalopsidae), where nasal tentacles have a mechanosensory function to ambush fish (Catania, Leiten, \& Gauthier, 2010). Among terrestrial snakes, horn-like cephalic appendages (hereafter, horns) are concentrated within the Viperidae, in which they are formed by either a single or multiple epidermal scales above the eyes (supraocular) or nasals (supranasal; Figure 1). Horns within the Viperidae are thus highly variable and occur in a variety of habitats across the globe, from sandy and rocky deserts (e.g., Bitis caudalis Smith, 1839) to tropical 
48 forests in terrestrial (e.g., Bitis rhinoceros Schlegel, 1855) and arboreal microhabitats (e.g.,

49 Atheris ceratophora Werner, 1896).

50 Early herpetologists have speculated that supraocular horns in the horned rattlesnake,

51 Crotalus cerastes Hallowell, 1854, may be of little evolutionary significance while others

52 suggested a protective role as an eyelid when snakes enter rodent burrows (Cohen \& Myres,

53 1970). These authors alluded to similar desert taxa inhabiting rodent burrows or rock crevices

54 but also proposed that the enlarged supraocular scales of arboreal taxa may serve a protective function among dense foliage. Wagner \& Wilms (2010) hypothesized that cephalic horns among vipers may serve a role in sexual recognition as in other squamates.

More recently, several hypotheses have been proposed in non-peer reviewed literature. Naish (2009) suggested horns may aid in camouflage of forest-living species by obscuring snakes' silhouette, but due to the diversity of cephalic appendages and wide range of ecologies horns may have evolved for multiple reasons. del Mármol (2020) supported the camouflage hypothesis in desert taxa and further speculated that horns may act as lures to attract rodent prey in sandy deserts or serve a protective role during prey capture. attention in recent scientific literature. Here, we assembled habitat and diet information on 263 viper taxa and use a phylogenetic comparative approach to shed light on the selection pressures underlying horn evolution. By assessing correlation between horns and habitat or and evolved repeatedly in similar environments. 
Methods

Data collection

72 As the basis of our comparative analyses, we used a previously published time-calibrated

73 phylogeny derived from six mitochondrial and five nuclear genes for 263 Viperidae taxa

74 (Alencar et al., 2016). For each taxon in the phylogeny, we assembled ecological and morphological trait data from published literature and online databases. The data was coded in a binary matrix, recording the presence/absence of a taxon under each variable. Habitat categories were defined by the IUCN habitat classification scheme (Version 3.1; https://www.iucnredlist.org/resources/habitat-classification-scheme), comprising forest, savanna, shrubland, grassland and desert habitats. We further recorded the presence in five microhabitat categories, including sandy (e.g., windblown sand dunes), rocky (e.g., outcrops, karst, cliffs), arboreal (including semi-arboreal), terrestrial forest (fully terrestrial taxa in forest habitat), and water-associated (e.g., swamps, marshes, rivers). We were able to obtain diet information for 224 out of the 263 taxa where we recorded the presence of six prey classes (mammals, reptiles, birds, amphibians, fish, and invertebrates) known from the diet of each taxon. Morphological traits were recorded from images on iNaturalist (https://www.inaturalist.org/), The Reptile Database (Uetz et al., 2021), or species descriptions for taxa where images were not available. We recorded the presence of ocular or nasal horns, defined as tapered projections above the eyes or rostral, respectively (Figure 1). We also identified intermediate characters that comprise either a pronounced ridge or comparatively smaller projections that do not taper to a point (Figure S1). We did not consider

91 dorsally flattened ocular ridges (e.g., large supraoculars of Crotalus spp. or Vipera spp.) to be intermediate character states of ocular horns. We were unable to account for intraspecific 
polymorphism of character states due to the binary nature of the correlation analyses present in a taxon. All binary statistical analyses were performed on two datasets. The 'horns' dataset comprises only the presence/absence of horns, and the 'characters' dataset comprises the presence/absence of horns or intermediate characters. In addition to analyzing the combined 'horns' and 'characters' datasets, we also analyzed nasal and ocular traits separately. All statistical analyses were conducted in R v.4.0.2 (R Core Team, 2020). The data underlying this work will be made available with the published version of the manuscript.

\section{Ancestral state reconstruction}

To determine the most parsimonious models of evolution, we fit various continuous-time Markov models to our datasets, using the 'fitMK' function in the R package phytools v.0.7.80 (Revell, 2012). For multiple states, e.g., ocular horns, nasal horns, or no horns, we fit equalrates (ER), symmetric-rates (SYM), and all-rates-different (ARD) models. Additionally, for the multistate datasets including horns and intermediate characters, we fit the ER, ARD and SYM models along with ordered versions of each model where a trait needs to transition to an using Akaike Information Criterion (AIC) and AIC weights (AICW). Ancestral state reconstruction of horn evolution was then estimated with the best-fit models (Table S1) using stochastic character mapping, simulating 1000 maps using the 'make.simmap' function in phytools. The root states (pi) of all models were estimated from the stationary distribution of the transition matrix $(\mathrm{Q})$ assuming a flat prior.

114 We tested for phylogenetic signal using $D$ statistics (Fritz \& Purvis, 2010) in the R package 
$116 D$ is equal to 1 if a binary trait is randomly distributed across the phylogeny, 0 if the trait is

117 phylogenetically clumped as if it had evolved by Brownian motion, and exceeds this range if

118 a trait is overly dispersed or more clumped than expected by Brownian motion (Fritz \& Purvis, 119 2010).

Habitat association

121 To test for correlations between association with habitat and the presence of 'horns' or 122 'characters', we employed two complementary approaches in phytools. First, we used the 123 'fitPagel' function that fits a model of correlated evolution between two binary traits (Pagel, 124 1994). This method uses a likelihood ratio test to compare two models in which binary traits either evolve independently or in a dependent manner. In our analyses trait states were set as the dependent variable and the "fitDiscrete" method was used to fit the model of discrete character evolution. Since vipers with ocular horns occur in terrestrial and arboreal habitats, we performed the analyses twice on each dataset: First on all taxa and then by removing the 75 arboreal taxa to identify terrestrial habitats where the selection pressures underlying horn evolution may be similar to arboreal microhabitats. The threshold for declaring statistical significance was set to $p<0.05$, and $p$-values were corrected for multiple comparisons using

\section{False Discovery Rate (FDR) correction.} and traits using a Bayesian version of the quantitative threshold model (Felsenstein, Ackerly, generations, sampling every 2000 , and discarding the first $20 \%$ of each chain as burn-in. We 
138 of direction (PD), an index of effect existence (Makowski et al., 2019). The effect is considered

139 significant if the entire 90\% HPD is either positive or negative, i.e., the PD > 95\%.

140 Relative diet specificity

141 First, we tested for correlated evolution between prey class and 'horns' or 'characters' using

142 'fitPagel' as above, which did not yield significant correlations (Table S2). Therefore, we

143 explored whether traits may be associated with specificity to a particular prey class using a

144 diet specificity index comparable to that of Maritz et al. (2016). We transformed the

145 presence/absence data to a relative diet specificity index (DSi) for each of the six prey classes,

146 which is presence (1) or absence (0) divided by the number of prey classes in the diet of a

147 taxon and ranked from zero. Thus, zero represents no consumption and higher rank

148 represents higher relative specificity to a particular prey class. Although this scaled index does

149 not provide an estimation of prey specificity within taxa, it can with the available data provide

150 a relative comparison among taxa. Only six taxa were recorded eating fish, therefore this prey

151 class was not included in further analyses. We tested for a correlation between traits and diet

152 specificity using phylogenetic generalized least squares (PGLS), implemented with residual

153 randomization in a permutation procedure in the RRPP package (Collyer \& Adams, 2018) with

15410000 permutations for significance testing. The multivariate response variables (DSi for five

155 prey classes) were evaluated using MANOVA and Pillai's trace test statistic, followed by

156 univariate ANOVAs to assess differences in specificity to each prey class. To test for dietary

157 differences between trait states associated with different habitats, we included an interaction

158 term between trait state and habitat. We specifically tested interaction among correlated

159 trait and habitat variables. 


\section{Results}

162 We identified 35 taxa out of 263 in which horns are present (13.3\%), 14 (5.3\%) with nasal

163 horns and 21 (8\%) with ocular horns (Figure 2). Additionally, we identified 33 taxa in which 164 intermediate character states were present (12.5\%), $22(8.4 \%)$ nasal and 11 (4.2\%) ocular. In 165 total we recorded 59 taxa (22.4\%) in which characters were present, 36 (13.7\%) with nasal 166 characters and $32(12.2 \%)$ with ocular characters. Of these, 9 taxa (3.4\%) can have both nasal 167 and ocular characters. The binary analyses were performed separately on the 'horns' dataset, 168 including only the presence/absence of horns (35 taxa), and on the 'characters' dataset, 169 including the presence of horns and intermediate characters (59 taxa).

The posterior distribution of 1000 stochastic character maps on the 'horns' dataset (Figure S2) estimated an average of 14 independent origins of ocular horns (95\% HPD: 12-16) with 1 reversal (95\% HPD: 0-3) and 11 origins of nasal horns (95\% HPD: 9-13) with 1 reversal (95\% HPD: 0-3). The posterior distribution on the 'characters' dataset (Figure S3) estimated 12 origins of ocular characters (95\% HPD: 10-15) and 4 reversals (95\% HPD: 1-7); 12 independent origins of nasal characters (95\% HPD: 9-14) with 2 reversals (95\% HPD: 0-4) and 3 transitions to a state with both characters (95\% HPD: 2-4). There was a mean of 4 independent origins of a state with both characters from a state with no characters (95\% HPD: 3-5).

179 For the multistate ocular and nasal datasets, the ordered models of evolution, where a trait needs to transition to an intermediate state before transitioning to the horned state, generally retrieved lower AIC values compared to the respective unordered models (Table 
followed by frequent transitions between intermediate characters and horns, and less estimated values of the $D$ statistic generally suggest stronger phylogenetic signal in the 'characters' datasets (characters: $D=-0.06$; ocular: $D=0.08$; nasal: $D=-0.25$ ) compared to the 'horns' datasets (horns: $D=0.33$; ocular: $D=0.16$; nasal: $D=0.18$ ). association evolve independently (Table S4). However, for both ocular and nasal horns the dependent models performed better where the rate of evolution was dependent on a taxon's association with terrestrial forest habitats (Tables $1 \&$ S4A). When arboreal taxa were removed to detect correlations among terrestrial habitats, the models where ocular horns depend on terrestrial forest, shrubland, rocky and desert habitats performed better compared to the respective independent models (Tables $1 \&$ S4A). Correlating the presence of horns with a taxon's occurrence in these habitats under the threshold model retrieved a moderate but significantly positive correlation between nasal horns and terrestrial forest habitats $(r=0.38$; PD $>95 \%$ ) while ocular horns had a moderate but significant negative correlation with terrestrial forest habitats ( $r=-0.45$; PD > 95\%; Figures 4 \& S6). After removing arboreal taxa, the correlations between ocular horns and occurrence in shrubland ( $r=0.35$; 
207 As with the 'horns' dataset, the correlation between ocular characters and terrestrial forest

208 was moderate but significantly negative ( $r=-0.39 ; \mathrm{PD}>95 \%$; Figure S7). In contrast, for nasal

209 characters, the dependent model of evolution was not significantly better than the

210 independent model (Table S4B) and assessing the correlation between forest habitats and

211 nasal characters under the threshold model revealed no significant correlation (Figure S7).

212 When arboreal taxa were removed, the rate of ocular character evolution was dependent on

213 the association with terrestrial forest, shrubland, rocky, and desert habitats, as well as sandy

214 habitat (Table S4B). Under the threshold model, the correlation between ocular characters

215 and terrestrial forest $(r=-0.41 ; P D>95 \%)$, shrubland $(r=0.31 ; P D>95 \%)$, rocky $(r=0.38 ; P D$

$216>95 \%$ ) and desert habitats ( $r=0.40$; PD > 95\%) were significant but the correlation with sandy

217 habitat was not significantly positive ( $r=0.30 ; 90 \%$ HPD: $-0.02-0.63$; Figure S7).

218 Taxa with nasal horns exhibit the highest shared association with terrestrial forest

219 microhabitats, 13 out of 14 taxa (92.9\%; Figure 2). Similarly, despite not detecting significant

220 correlations, the highest shared habitat association among taxa with nasal characters is with

221 forest habitat (33/36; 91.6\%), followed by terrestrial forest microhabitats $(24 / 36 ; 66.6 \%)$.

222 Only one out of 21 taxa with ocular horns is associated with terrestrial forest microhabitats

223 (4.5\%), reflecting shared dissociation. Among the habitats correlated with ocular horns, the

224 highest shared association is with rocky microhabitats $(15 / 21 ; 71.4 \%)$, followed by shrubland

$225(12 / 21 ; 57,1 \%)$, desert $(8 / 21 ; 38.1 \%)$, arboreal $(8 / 21 ; 38.1 \%)$ and sandy $(6 / 21 ; 28.6 \%)$. This

226 trend is consistent for taxa with ocular characters (Figure 2).

227 Relative diet specificity

228 The presence of horns or characters did not have a significant effect on relative diet 229 specificity, nor did the different horn or character types (Tables S5A \& S6A). The interaction 
terms between traits and habitat generally did also not have significant effects on relative univariate effects (Tables S5 \& S6). The interaction term between arboreal microhabitats and ocular traits did have a significant effect on relative specificity to invertebrate prey. Arboreal taxa with ocular traits had significantly lower relative specificity compared to arboreal taxa without ocular traits (Tables S5F \& S6E). There are, however, only six out of 75 arboreal taxa recorded feeding on invertebrates (although none with ocular traits), thus this result should be interpreted with caution. The interaction term between ocular horns and desert habitat had a significant multivariate effect on relative diet specificity, where diet specificity of taxa with ocular horns associated with deserts is significantly different from taxa with ocular horns not associated with deserts (Table S5G). This interaction term also had a significant univariate effect on relative specificity to reptile prey, with no significant pairwise differences, as well as a significant effect on relative specificity to bird prey, where taxa with ocular horns in deserts had significantly higher specificity compared to all other groups (Table S5G). However, in the 'characters' dataset the interaction term between desert and ocular characters had a significant effect only on relative specificity to bird prey, where taxa with ocular characters in deserts have significantly higher specificity compared to taxa with ocular characters not associated with deserts (Table S6F).

There is a notable difference in the proportion of taxa with each trait that have been recorded feeding on amphibians (Figure S8). $78.6 \%$ and $69.4 \%$ of taxa with nasal horns and characters were recorded feeding on amphibians compared to only $28.6 \%$ and $31.3 \%$ of taxa with ocular characters, while the proportion of taxa feeding on other prey classes were comparable. This reflects the general occurrence of taxa with nasal horns in mesic forest environments compared to taxa with ocular horns in arid environments. 
254

255

256

257

258

259

260

261

262

263

\section{Discussion}

Our analyses revealed significant correlations between the evolution of horns and habitat despite the low frequency of Viperidae taxa in which horns and intermediate characters are present. The relative positions of horns are ecologically divergent in that taxa with nasal characters are associated with terrestrial forest and forest habitats while taxa with ocular characters are dissociated with terrestrial forest habitats and associated with arboreal or sparsely vegetated terrestrial habitats (Figures $2 \& 4$ ). Furthermore, multiple independent origins of similar traits in similar habitats across the globe provides evidence of adaptive convergence.

Multiple origins and transitions between character states suggest that cephalic horns are highly labile within the Viperidae. Frequent transitions, particularly between intermediate character states and horns (Figure 3), fit a theoretical model of evolution under Brownian motion whereby a continuously varied trait fluctuates randomly over time (Revell, Harmon, \& Collar, 2008). This observation is supported by significant Brownian phylogenetic structure across all datasets (Table S3). Evolution by Brownian motion does not necessarily imply random drift. Instead strong phylogenetic signal and oscillation in the extent of character states can also be explained by fluctuating selection in variable environments over time or among populations (O'Meara et al., 2006; Revell et al., 2008; Bell, 2010). In this regard, reduced rate of fluctuating selection, stabilizing or divergent selection can decrease the strength of phylogenetic signal (Revell et al., 2008). Therefore, the ordered models of evolution along with a reduction in phylogenetic signal between the 'characters' and 'horns' datasets may suggest that horns evolved under selection from an intermediate state. Furthermore, the non-random phylogenetic clumping of ocular and nasal characters in 
particular habitat types suggest that characters are selected for in the habitats with which

they associate or selected against in the habitats with which they do not associate while

evolving randomly in other habitats, or both (Figure 2). Correlations with habitat make it

tempting to assume that the observed patterns of horn evolution was driven by frequent

habitat shifts throughout the planet's dynamic history. It should, however, be noted that

vipers, including polymorphic horned taxa, may exhibit substantial phenotypic variability

among and within populations (Wagner \& Wilms, 2010; Pizzigalli et al., 2020). It is therefore

likely that microevolutionary processes at the population level contribute to the frequent

transitions of character states observed at a macroevolutionary scale (Li et al., 2018).

Significant correlations between horns and habitat may not be sufficient to determine

causation of horn evolution in vipers, especially since $<13.3 \%$ of the vipers in our analyses

possess horns, although habitat use has been attributed to distant morphological

convergence in other snake families (Grundler \& Rabosky, 2014; Esquerré \& Scott Keogh,

expected. As ambush predators vipers are generally opportunistic with wide diet breadths

(Glaudas et al., 2019), thus diet is likely to be constrained mainly by available prey in the environment without limiting morphological convergence (Grundler \& Rabosky, 2014). This notion is evident in the PGLS where habitat had the largest effect on relative diet specificity

with deserts and ocular characters not associated with desert habitat (Tables S5G \& S6F). 
foraging. Nasal appendages occur in Ahaetulla (Colubridae) and Langaha (Lamprophiidae) species, two distantly related arboreal genera thought to rely on crypsis to ambush diurnal prey (Henderson \& Binder, 1980; Kuchling, 2003; Tingle, 2012; Mallik et al., 2020). Two of the three Langaha species possess both nasal and ocular appendages (Kuchling, 2003), which is comparable to the arboreal viper taxa we recorded with both characters (Figure 2). Nasal appendages also occur in two arboreal forest-living Gonyosoma (Colubridae) species (Peng et al., 2021). Information on the foraging behavior of this genus is limited but they may use ambush and active foraging modes (Hodges, 2019). In non-arboreal habitats, Trachyboa boulengeri Peracca, 1910 (Tropidophiidae) possesses both characters, is highly cryptic and utilizes an ambush technique to prey on fish and frogs (Dieter Lehmann, 1969; Arteaga, 2021). Ambush foraging has also been observed in the horned sea snake, Hydrophis peronii Duméril, 1853 (Elapidae; Borsa, 2008). Acanthophis (Elapidae), in which some species have horn-like supraocular scales, share remarkable morphological convergence with terrestrial vipers and like many vipers also employ caudal luring to attract prey (Shine, Spencer, \& Keogh, 2014; Crowe-Riddell et al., 2021). These comparisons may support the hypothesis that horns evolved under selection for crypsis in ambush foraging snakes.

Crypsis is highly dependent on habitat and hunting location of predators, which often results in different modes of camouflage among related organisms in forested compared to open habitats (Pembury Smith \& Ruxton, 2020; Caro \& Koneru, 2021). This trend is apparent in the Viperidae, especially among Bitis species where terrestrial forest taxa possess nasal horns and open habitat taxa possess ocular horns (Figures 1, 2 \& 4). In most cases cryptic or disruptive colors are sufficient forms of camouflage (Egan et al., 2016; Hughes, Liggins, \& Stevens, 2019). Yet, there may be scenarios where disruption or deception by secondary appendages could reduce detection and increase fitness. For example, over evolutionary time predator or prey 
could adapt to the camouflage strategies of the other and thereby drive the evolution of

325 improved crypsis (Pembury Smith \& Ruxton, 2020). Crypsis can also evolve to enhance diurnal

326 concealment or to the differential visual systems of specific organisms (Hughes et al., 2019;

327 Caro \& Koneru, 2021).

Comparisons between vipers, other snakes and other forest-living squamates (Losos et al.,

2012; Breuil et al., 2019; Karunarathna et al., 2020) suggests a broader convergence of nasal

appendages in forest-associated taxa. Terrestrial forest habitats are typically covered in a

layer of leaf litter or foliage which terrestrial vipers utilize as concealment to ambush prey.

Here, nasal horns could potentially disrupt the outline of a viper's head, making it more

difficult to detect against a background of leaves and twigs. In contrast, vipers in arboreal,

rocky, or sparsely vegetated open habitats may be more limited to exposed ambush sites with

little cover. It has previously been hypothesized that the vertical pupils of ambush foraging

snakes aid in concealment by disrupting the round outline of the eye (Brischoux, Pizzatto, \&

Shine, 2010). The position of supraocular horns would contribute significantly to concealing

the eyes and disrupting a viper's silhouette in more exposed ambush sites. In this regard, the

selection pressures imposed by the arboreal ambush sites of Bothriechis schlegelii Berthold,

1846 may be analogous to the arid rocky habitats of Pseudocerastes species. Both these taxa

are able to rely on crypsis to ambush diurnal prey while avoiding predation (Sorrell, 2009; del

Mármol, Mozaffari, \& Gállego, 2016). Similarly, two ocular horned vipers occurring at high

343 altitudes, Mixcoatlus melanurus Müller, 1924 and Ophryacus undulatus Jan, 1859, are

344 thought to be constrained to diurnal foraging habits due to low nocturnal temperatures

345 (O’Shea, 2018). The occurrence of supraocular horns in cryptic lizard genera, e.g.,

346 Acanthosaura and Lyriocephalus (Agamidae) as well as Uroplatus (Gekkonidae), may further

347 support their role in crypsis (Rodda, 2020). 
348 While our analyses provide evidence to support convergent evolution of Viperidae horns in

349 similar habitats, more detailed studies are needed to fully understand their role in crypsis.

350 The relationship between horns and camouflage may be complex, especially among variable

351 populations and/or environments where there are likely to be community dependent

352 tradeoffs between hunting success and predator avoidance.

\section{Acknowledgments}

354 We thank Adriaan Jordaan, Courtney Hundermark, Tyrone Ping and Sterrin Smalbrugge for

355 their assistance with the literature review, and Bryan Maritz for sharing diet data. We are

356 grateful to Sandra Goutte for suggestions on the analyses, and Sebastian Kirchhof and Justin

357 Wilcox for comments on the manuscript. This work was supported in part by the NYU Abu

358 Dhabi Global PhD Student Fellowship. 


\section{References}

360

361

362

Alencar LRV, Quental TB, Grazziotin FG, Alfaro ML, Martins M, Venzon M \& Zaher H. 2016. Diversification in vipers: Phylogenetic relationships, time of divergence and shifts in speciation rates. Molecular Phylogenetics and Evolution 105: 50-62.

Arteaga A. 2021. Northern Eyelash-Boa (Trachyboa boulengeri). In: Arteaga A, Bustamante L, Vieira J, Guayasamin J, eds. Reptiles of Ecuador: Life in the middle of the world. Available at: https://www.tropicalherping.com

Bell G. 2010. Fluctuating selection: The perpetual renewal of adaptation in variable environments. Philosophical Transactions of the Royal Society B: Biological Sciences 365: 87-97.

Bergmann PJ \& Berk CP. 2012. The evolution of positive allometry of weaponry in horned lizards (Phrynosoma). Evolutionary Biology 39: 311-323.

Borsa P. 2008. Sea snakes: Overlooked predators at an urban fringing reef. Coral Reefs 27: 743.

Breuil M, Vuillaume B, Schikorski D, Krauss U, Morton MN, Haynes P, Daltry JC, Corry E, Gaymes G, Gaymes J, Bech N, Jelić M \& Grandjean F. 2019. A story of nasal horns: Two new subspecies of Iguana Laurenti, 1768 (Squamata, Iguanidae) in Saint Lucia, St Vincent \& the Grenadines, and Grenada (southern Lesser Antilles). Zootaxa 4608: 201-232.

Brischoux F, Pizzatto L \& Shine R. 2010. Insights into the adaptive significance of vertical pupil shape in snakes. Journal of Evolutionary Biology 23: 1878-1885.

Caro T \& Koneru M. 2021. Towards an ecology of protective coloration. Biological Reviews 96: 611-641.

Catania KC, Leiten DB \& Gauthier D. 2010. Function of the appendages in tentacled snakes (Erpeton tentaculatus). Journal of Experimental Biology 213: 359-367.

Cohen AC \& Myres BC. 1970. A function of the horns (supraocular scales) in the sidewinder rattlesnake, Crotalus cerastes, with comments on other horned snakes. Copeia 1970: 574.

Collyer ML \& Adams DC. 2018. RRPP: An r package for fitting linear models to high-dimensional data using residual randomization. Methods in Ecology and Evolution 9: 1772-1779. 
385 Sanders KL \& Allen L. 2021. From matte banded to glossy black: Structures underlying colour change in the caudal lures of southern death adders (Acanthophis antarcticus, Reptilia: Elapidae). Biological Journal of the Linnean Society 132: 666-675.

Dieter Lehmann H. 1969. Beobachtungen bei der Haltung und Aufzucht von Trachyboa boulengeri (Serpentes, Boidae). Salamandra 6: 32-42.

Egan J, Sharman RJ, Scott-Brown KC \& Lovell PG. 2016. Edge enhancement improves disruptive camouflage by emphasising false edges and creating pictorial relief. Scientific Reports 6: 38274.

Esquerré D \& Scott Keogh J. 2016. Parallel selective pressures drive convergent diversification of phenotypes in pythons and boas. Ecology Letters 19: 800-809.

Felsenstein J, Ackerly DD \& Mcpeek MA. 2012. A comparative method for both discrete and continuous characters using the threshold model. American Naturalist 179: 145-156.

Fritz SA \& Purvis A. 2010. Selectivity in mammalian extinction risk and threat types: A new measure of phylogenetic signal strength in binary traits. Conservation Biology 24: 1042-1051. of Animal Ecology 88: 757-767.

Grundler MC \& Rabosky DL. 2014. Trophic divergence despite morphological convergence in a continental radiation of snakes. Proceedings of the Royal Society B: Biological Sciences 281: 20140413.

Henderson RW \& Binder MH. 1980. The ecology and behavior of vine snakes: a review. Milwaukee Public Museum Contributions in Biology and Geology 37: 1-38. 801. 

1079-1088. patterns. BMC Evolutionary Biology 18: 123.

Losos JB. 2011. Convergence, adaptation, and constraint. Evolution 65: 1827-1840.

Herrel A. 2012. Notes on the natural history of the little-known Ecuadorian horned anole, Anolis proboscis. Breviora 531: 1.

Mahler DL, Weber MG, Wagner CE \& Ingram T. 2017. Pattern and process in the comparative study of convergent evolution. American Naturalist 190: S13-S28.

Makowski D, Ben-Shachar MS, Chen SHA \& Lüdecke D. 2019. Indices of effect existence and significance in the

Mallik AK, Srikanthan AN, Pal SP, D’Souza PM, Shanker K \& Ganesh SR. 2020. Disentangling vines: a study of 429 morphological crypsis and genetic divergence in vine snakes (Squamata: Colubridae: Ahaetulla) with the 430 description of five new species from Peninsular India. Zootaxa 4874: 1-62. 
434 del Mármol GM. 2020. The phenotypic variability of the genus Bitis Gray 1842, with remarks in its resemblance

435 to other vipers. In: Martínez G, León R, Jiménez-Robles O, González De la Vega JP, Gabari V, Rebollo B, Sánchez-

436 Tójar A, Fernández-Cardenete JR, Gállego J, eds. Amphibians and Reptiles of Morocco and Western Sahara.

437 Available at: http://www.moroccoherps.com

del Mármol GM, Mozaffari 0 \& Gállego J. 2016. Pseudocerastes urarachnoides: the ambush specialist. Boletín

de la Asociación Herpetológica Española 27: 36-42.

440 Naish D. 2009. Why do some snakes have horns? ScienceBlogs. Available at: https://scienceblogs.com

441 O’Meara BC, Ané C, Sanderson MJ \& Wainwright PC. 2006. Testing for different rates of continuous trait 442 evolution using likelihood. Evolution 60: 922.

O’Shea M. 2018. The book of snakes: A life-size guide to six hundred species from around the world. University of Chicago Press.

Orme D, Freckleton R, Thomas G, Petzoldt T, Fritz S, Isaac N \& Pearse W. 2013. The caper package: comparative analysis of phylogenetics and evolution in R. R package version 5: 1-36.

Pagel M. 1994. Detecting correlated evolution on phylogenies: A general method for the comparative analysis of discrete characters. Proceedings of the Royal Society B: Biological Sciences 255: 37-45.

Pembury Smith MQR \& Ruxton GD. 2020. Camouflage in predators. Biological Reviews 95: 1325-1340.

Peng LF, Zhang Y, Huang S, Burbrink FT, Chen JM, Hou M, Zhu YW, Yang H \& Wang JC. 2021. A new snake species of the genus Gonyosoma Wagler, 1828 (Serpentes: Colubridae) from Hainan Island, China. Zoological

Pizzigalli C, Banfi F, Ficetola GF, Falaschi M, Mangiacotti M, Sacchi R, Zuffi MAL \& Scali S. 2020. Ecogeographical determinants of the evolution of ornamentation in vipers. Biological Journal of the Linnean Society 130: $345-358$.

R Core Team. 2020. R: a language and environment for statistical computing. Vienna: $R$ Foundation for Statistical

457 Computing. Available at: https://www.R-project.org 
459 Ecology and Evolution 3: 217-223.

460 Revell LJ, Harmon L \& Collar DC. 2008. Phylogenetic signal, evolutionary process, and rate. Systematic biology

$461 \quad 57: 591-601$.

462 Rodda GH. 2020. Lizards of the world: Natural history and taxon accounts. Baltimore: Johns Hopkins University

463 Press.

464 Shine R, Spencer CL \& Keogh JS. 2014. Morphology, reproduction and diet in Australian and papuan death

465 adders (Acanthophis, elapidae). PLOS ONE 9: 94216.

466 Sorrell GG. 2009. Diel movement and predation activity patterns of the eyelash Palm-pitviper (Bothriechis

467 schlegelii). Copeia 2009: 105-109.

468 Stuart-fox DEVI. 2013. Chameleon behavior and color change. In: Tolley KA, Herrel A, eds. The biology of

469 chameleons. University of California Press, 115-130.

470 Tingle JL. 2012. Field observations on the behavioral ecology of the Madagascan Leaf-Nosed Snake, Langaha

471 madagascariensis. Herpetological Conservation and Biology 7: 442-448.

472 Uetz P, Freed P, Aguilar R \& Hošek J. 2021. The reptile database. Available at: http://www.reptile-database.org

473 Wagner P \& Wilms TM. 2010. A crowned devil: new species of Cerastes Laurenti, 1768 (Ophidia, Viperidae) from

474 Tunisia, with two nomenclatural comments. Bonn zoological Bulletin 57: 297-306. 


\section{Supporting information}

476 Table S1. Best-fit continuous-time Markov models.

477 Table S2. 'fitPagel' results on diet classes.

478 Table S3. Phylogenetic signal, $D$ statistics.

479 Table S4. 'fitPagel' results on habitat.

480 Table S5. Phylogenetic generalized least squares on relative diet specificity. Horns dataset.

481 Table S6. Phylogenetic generalized least squares on relative diet specificity. Characters

482 dataset.

483 Figure S1. Examples of intermediate cephalic characters in the Viperidae.

484 Figure S2. Ancestral state reconstruction of cephalic horns in Viperidae.

485 Figure S3. Ancestral state reconstruction of cephalic characters in Viperidae.

486 Figure S4. Ancestral state reconstruction of nasal horns in Viperidae.

487 Figure S5. Ancestral state reconstruction of ocular horns in Viperidae.

488 Figure S6. Posterior estimate of the correlation coefficient $(r)$ between the presence of horns 489 and habitat.

490 Figure S7. Posterior estimate of the correlation coefficient $(r)$ between the presence of 491 characters and habitat.

492 Figure S8. Relative proportion of Viperidae taxa with cephalic traits recorded feeding on each 493 prey class. 
Table 1. $\log$-likelihood $(\log \mathrm{L})$ for models of evolution between habitat and horns within the Viperidae. Showing $p$-values and FDR corrected $p$-values for independent models of evolution based on the likelihood ratio test. Significant p-values shown in bold. Complete results on all datasets are available in Table S4.

\begin{tabular}{|c|c|c|c|c|c|c|c|c|}
\hline \multirow[b]{2}{*}{ Habitat } & \multicolumn{4}{|c|}{ Nasal horns } & \multicolumn{4}{|c|}{ Ocular horns excluding arboreal taxa } \\
\hline & $\begin{array}{c}\text { Independent } \\
\log \mathrm{L}\end{array}$ & $\begin{array}{c}\text { Dependent } \\
\text { logL }\end{array}$ & p-value & $\begin{array}{c}\text { Corrected } \\
\text { p-value }\end{array}$ & $\begin{array}{c}\text { Independent } \\
\log \mathrm{L}\end{array}$ & $\begin{array}{c}\text { Dependent } \\
\text { logL }\end{array}$ & p-value & $\begin{array}{c}\text { Corrected } \\
\text { p-value }\end{array}$ \\
\hline forest & -164.508 & -163.599 & 0.403 & 0.832 & - & - & - & - \\
\hline terrestrial forest & -195.466 & -190.466 & 0.007 & $0.067^{*}$ & -144.187 & -136.615 & 0.001 & 0.004 \\
\hline arboreal & -112.551 & -112.551 & 1.000 & 1.000 & - & - & - & - \\
\hline savanna & -148.534 & -148.061 & 0.623 & 0.832 & -124.588 & -124.194 & 0.675 & 0.771 \\
\hline shrubland & -198.307 & -197.329 & 0.376 & 0.832 & -151.070 & -145.660 & 0.004 & 0.012 \\
\hline grassland & -188.919 & -188.512 & 0.666 & 0.832 & -153.892 & -152.360 & 0.216 & 0.288 \\
\hline sandy & -125.469 & -125.011 & 0.633 & 0.832 & -110.996 & -108.243 & 0.064 & 0.102 \\
\hline rocky & -188.286 & -187.357 & 0.395 & 0.832 & -146.751 & -140.937 & 0.003 & 0.012 \\
\hline desert & -125.146 & -125.146 & 1.000 & 1.000 & -114.829 & -110.771 & 0.017 & 0.035 \\
\hline water-associated & -125.146 & -200.215 & 0.490 & 0.832 & -138.649 & -138.649 & 1.000 & 1.000 \\
\hline
\end{tabular}

*Corrected $\mathrm{p}$-value not significant at alpha $=0.05$ but correlation under the threshold model is significant (Figure 4 ). 
496 Figure 1. Diversity of cephalic appendages in the Viperidae. Showing examples of taxa with supranasal (nasal) and supraocular (ocular) horns along with the broad geographic region where these taxa occur. Images sourced from Adobe Stock.

Figure 2. Evolutionary history of cephalic horns in the Viperidae along with correlated habitat variables. Branches are colored to show the character states estimated with the highest probability at each node. Exact trait probabilities are available in Figures S2-S5. Branch colors prioritize the full horn character states if present. Shading of taxon labels depict the character states of each taxon and the binary matrix indicates the association with each habitat variable.

Bar graphs show the relative proportion of taxa with each trait associated with the correlated habitat variables compared to the proportion of taxa with the trait not associated with each habitat. Values in the columns depict the absolute number of taxa. Note that 'characters'

507 include horns and intermediate character states.

Figure 3. Mean number of transitions between nasal (A) and ocular (B) character states estimated from the posterior distribution of 1000 stochastic character maps (Figures S4 \& S5).

$51095 \%$ highest posterior density interval shown in parentheses.

511 Figure 4. Contrasting correlation between horns and terrestrial forest habitat. Showing 512 posterior estimate of the correlation coefficient $(r)$ between the presence of nasal or ocular 513 horns and terrestrial forest habitat under the threshold model. Dot indicates the mean of the 514 posterior distribution. Error bars indicate the $90 \%$ highest posterior density, i.e., the 515 probability of direction is $>95 \%$. 


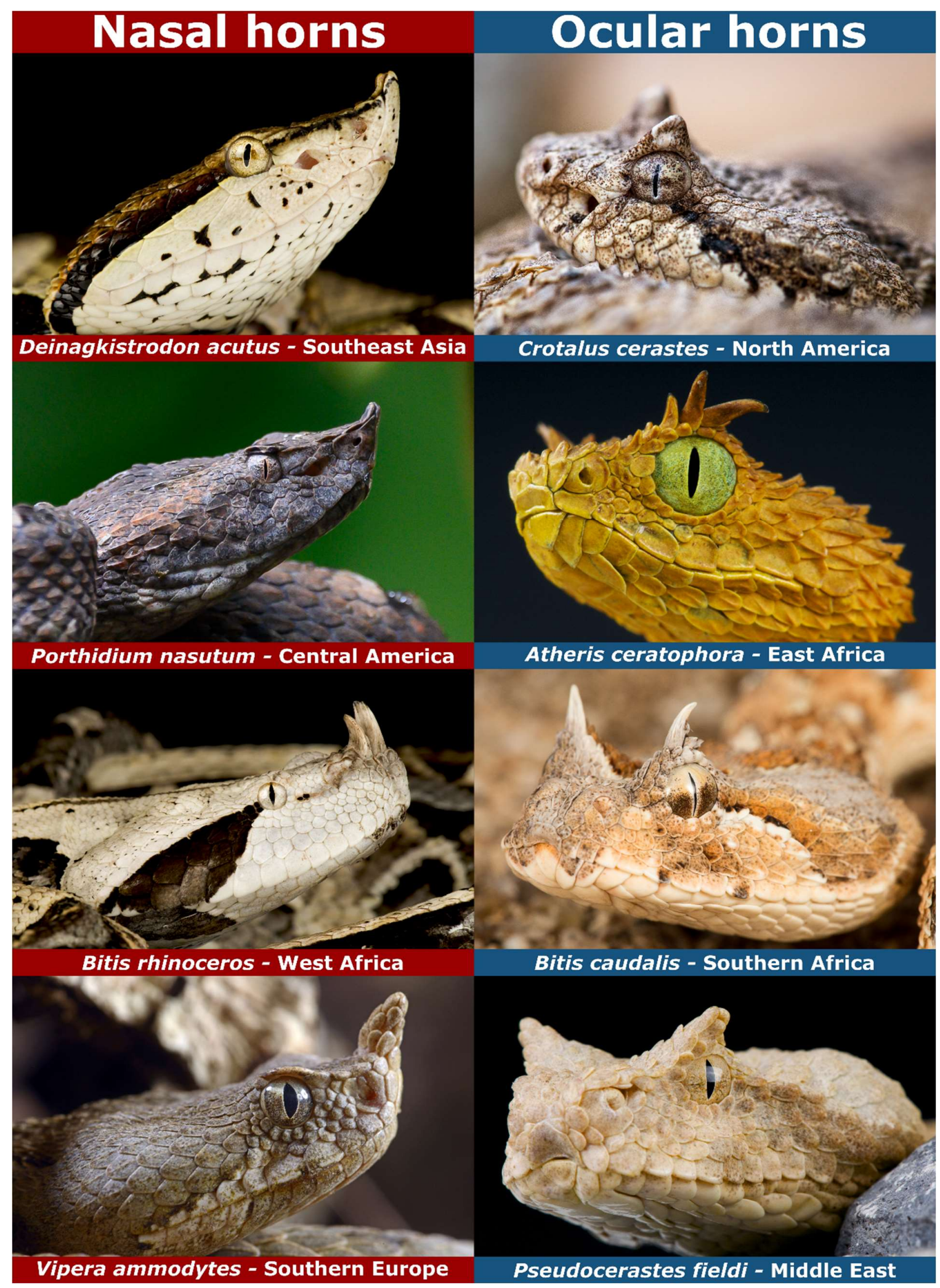


bioRxiv preprint doi: https://doi.org/10.1101/2021.10.24.465115; this version posted October 24, 2021. The copyright holder for this preprint (which was not certified by peer review) is the author/funder, who has granted bioRxiv a license to display the preprint in perpetuity. It is made available under aCC-BY-NC-ND 4.0 International license.
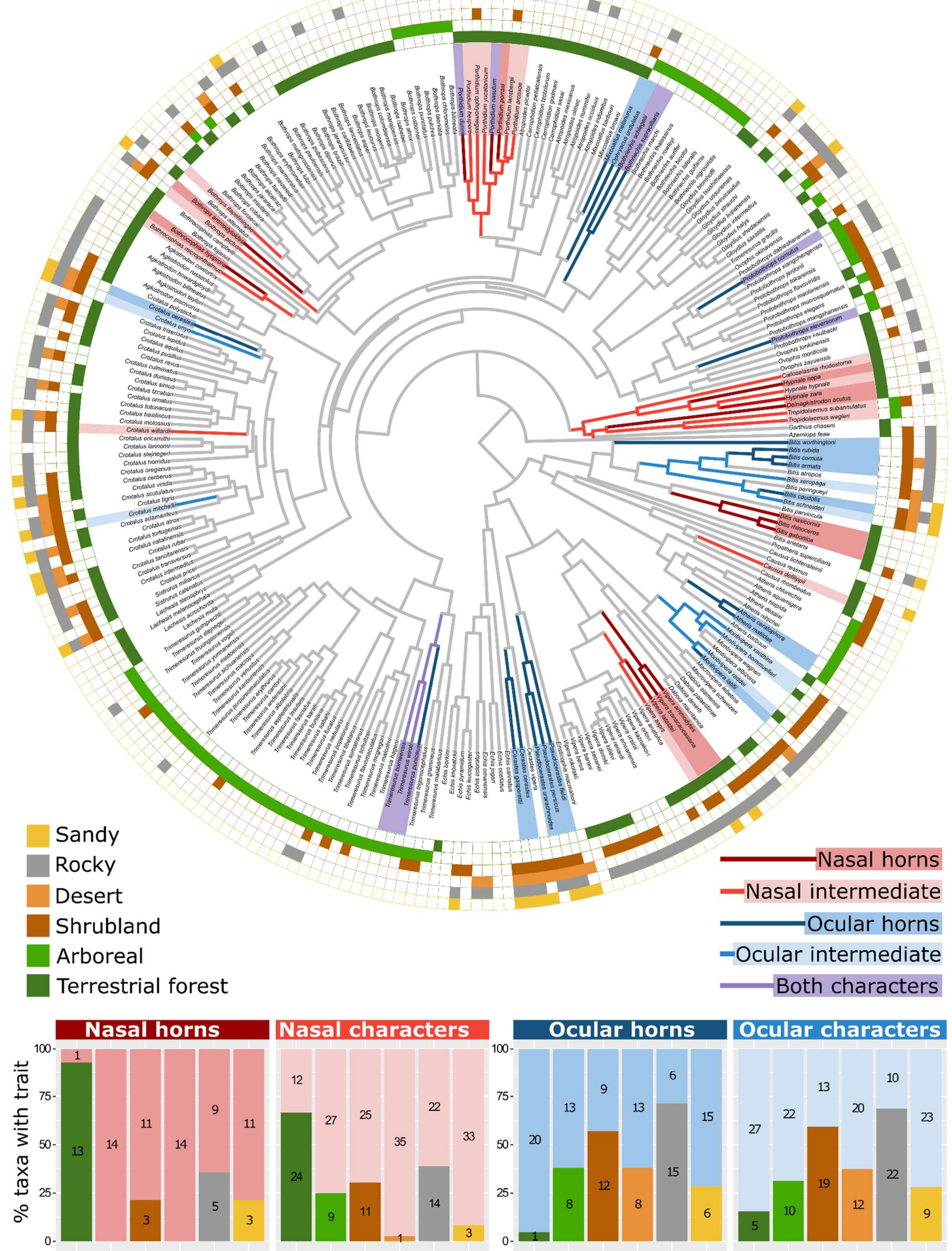

Ocular characters

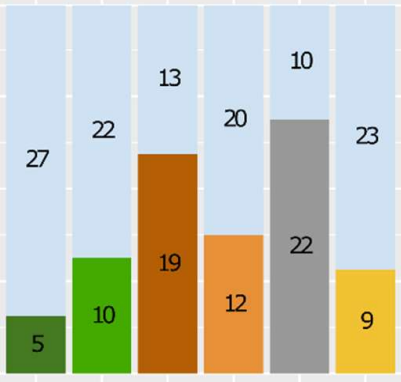

Figure 2. 


\section{A. No nasal}

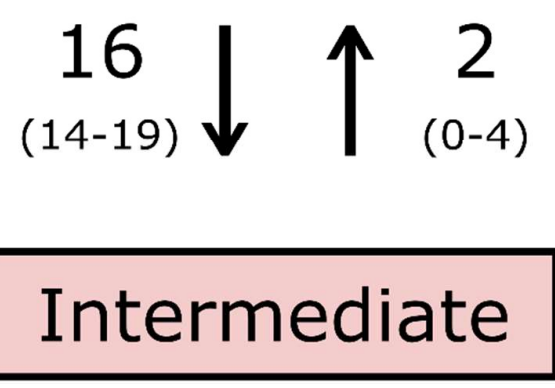

\section{B. No ocular}

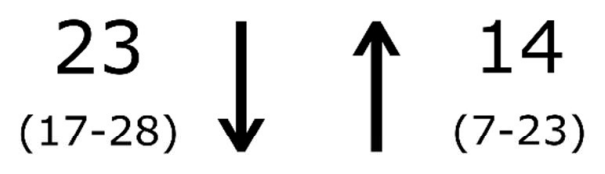

\section{Intermediate}

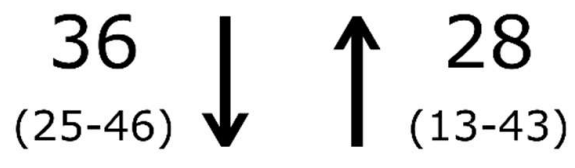

\section{Nasal horns}

\section{Ocular horns}

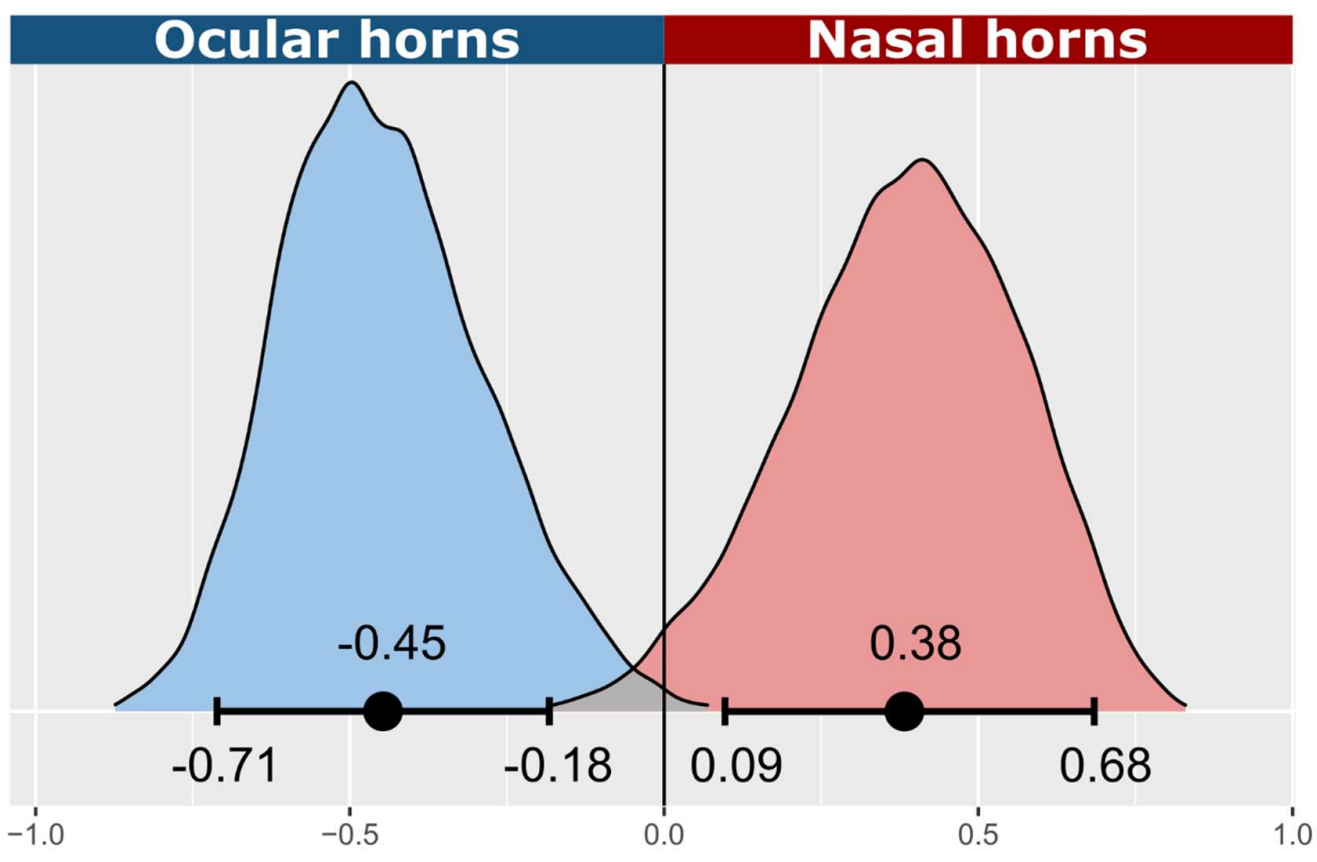

